\title{
Live attenuated vaccine trials in medically informed volunteers: a special case?
}

Anthony J Pinching St Bartholomew's and The Royal London School of Medicine and Dentistry, London

\begin{abstract}
A group of activist clinicians have offered to volunteer for clinical trials of live attenuated HIV vaccines. This has provided an important conceptual challenge to medical ethics, and to work on the development of HIV vaccines. In exploring these issues, this article highlights how the HIV field has altered the content as well as the tone of ethical discourse. The balance of expertise and authority between research subjects and triallists is profoundly changed, raising questions about the limits of voluntarism and differing perspectives on risk-benefit analysis. Care is needed to ensure that the novelty of the situation does not confuse the central ethical and scientific issues.

(Fournal of Medical Ethics 2000;26:44-46)

Keywords: HIV vaccines; research ethics; risk-benefit analysis; voluntarism; consent
\end{abstract}

HIV vaccine research has proved as frustrating as its necessity is vital. The technical and conceptual problems of vaccine development are at least as challenging as the ethical debates regarding their trials and potential application, as seen in the article by Guenter et al. ${ }^{1}$ In recent years, a group of activist clinicians, some of whom are self-declared members of at-risk communities and/or are involved in clinical care of people with HIV infection, have offered themselves as volunteers for trials of live attenuated vaccines. This has raised some intriguing new slants on some key ethical issues.

The technical background is simply enough stated. Some of the most effective vaccines in the past have been live, but attenuated, strains of the virulent organisms against which they are able to confer protection. They provide better protection because, by replicating in the host, they can stimulate a more substantial and broadly based immune response. Such vaccines are based on strains of organisms that have been passaged in culture or through other species and have been shown not to revert to the "wild-type", diseaseproducing strain. There are some, albeit provisional, data to suggest that some strains of HIV may be less pathogenic. The proposal is that these well-informed volunteers would be willing to act as guinea pigs in the evaluation of such vaccines.

This generous, but also provocative, offer raises complex issues about process in research ethics, risk-benefit analysis, consent, disclosure, voluntarism, expertise and the duty of care, as well as concerns about timeliness, evaluability and the hazards to research processes and opportunities in the event of failure.

Firstly, we need to look at the very special kind of volunteers who are putting themselves forward. Arguably, they are among the best-informed people in the field: they have substantial clinical experience of the disease against which protection is being sought, and they are, in a number of instances, members of communities that have been substantially affected by HIV, giving them a keen personal sense of its community impact. However, does this very proximity undermine or influence adversely the principle of voluntarism? Are they in effect being implicitly or unconsciously coerced by their professional and/or community awareness of the issues? Idealism and solidarity are evident, but is there a sense of duty arising from their awareness of the limitations of medicine, from the burden of grief for patients or their peer-groups, or even an element of guilt at having escaped the fate of their patients and friends? Can we regard doctors in this field as somehow distinct from others who may volunteer and if so how? Do they need more or less protection from the influence of their prior perceptions? In pressing for these studies to be done and to be done on them, how do they affect the issue of equipoise that should inform study acceptability and design?

The offer to be trial subjects from individuals who are so highly informed seems to turn upside down much of the ethical literature about disclosure and consent to clinical trials. The presumption in most of it is that the doctor conducting the trial is substantially more informed than the trial participant. If the situation is reversed, then how does this affect the relative roles of the researcher conducting the trial - the triallist, and the volunteer? If the informed volunteer urges the 
triallist to proceed and to accept the trial risks that the volunteer has accepted, should the triallist defer totally, or does he/she still have to concur? Who has the power of veto on grounds of ethical acceptability? It has been said that "professionals should not set the moral agenda for their relationship with those they serve", ${ }^{2}$ but what about vice versa? Can the volunteer set the moral agenda of the acceptability of such a trial for the triallist? And who carries moral or legal responsibility in the event of problems? Surely, even where volunteers are better informed than the triallist, their role as participants means that they cannot resolve the competing roles of supervising the research and participating in it? Good medical ethics is said to demand "shared decision making", ${ }^{2}$ but this seems to be a particular example where the balance is almost too close for comfort!

\section{Duty of care}

Who in this setting is the "expert"? The volunteer could claim the expertise to define the limits of acceptability of a trial on ethical grounds, given that it is he who is consciously and knowledgeably taking the risk. The volunteer could argue that it is his assessment of the risk-benefit equation that must prevail. But should the triallist take a "paternalistic" role because of his obligation as the person who must take responsibility for trial conduct, including its ethics? What are the limits of beneficence in agreeing to the self-determination of a willing and well-informed volunteer? Whose perception of risk takes precedence? Whose "conflict of interest" should prevail? The clinical investigator surely still has a duty of care to ensure that trial participation does not create hazard for the patient, as well as enabling the best research opportunities to be taken.

Precipitateness is another issue in this unusual setting. The volunteers have put themselves forward in a way that could be construed as putting pressure on vaccine triallists to undertake such a study. That may indeed have been, in part, their intent. The urgency is real enough. Yet that urgency has been present for well over a decade. Pressure to take some bold steps, even short cuts, is certainly fuelled by the lack of progress with viral protein vaccines. Is there also a sense of global solidarity because of the increasing disparity between what can be offered as treatment to those in the industrialised, and to those in the developing, world? These arguments would appear to justify proceeding apace on the grounds of the ethics of emergency.

The balance in favour of attempting the live attenuated approach may have been tipped by the impressive effectiveness of combination anti- retroviral therapy. This could have been seen as providing something of a safety net for the vaccinees, if the vaccine strains proved to be, or reverted to, pathogenic strains. Is the urgency to proceed based on the need and on a perception of the available data, rather than its realistic appraisal? Can we genuinely identify nonpathogenic strains? Is there a sufficiency of data now to justify the view that immune protection can indeed be provided, or are we still too unclear about the nature, correlates and induction of such protection? Is a false sense of security being provided by the partial data that we have to date?

Yet one could argue that there will always be uncertainties, always more data to be gathered, but that ultimately the only way forward is to try something that we know from other settings to be the most likely way of achieving an effective vaccine. While some data can be obtained from animal experiments including those on primates, the reality is that HIV is a uniquely human pathogen. Therefore it is only in the setting of clinical trials that we are going to find out the vital answers. So why not just try it, if there are willing guinea pigs? The argument is an appealing offer to break the gridlock of incomplete and unsatisfactory knowledge derived from only partially relevant extrapolations. After all, Jenner didn't wait for the laboratory data to stack up before applying the original vaccine against smallpox. (However, Jenner did have a raft of human experience, almost literally "in the field", to draw upon!)

If we do look at the available and relevant data, can we draw any conclusions? The Sidney cohort of transfusion recipients is a key group that can offer insights. ${ }^{3}$ In this group of transfusion recipients, and the relevant donor, the strain infecting them has a deletion in the nef gene. Their clinical course seemed to be non-progressive over a long period of observation. Primate studies had also indicated that deletions in the nef gene could render the virus less pathogenic in a model system. ${ }^{4}$ These two strands of evidence seemed to provide encouragement.

\section{Timely reminder}

However, the clinical data from Sidney are necessarily time-limited, and more recent studies on the cohort ${ }^{3}$ have indeed started to suggest that there is evidence of disease progression, or immune decline, after longer periods of observation. This is a timely reminder of the importance of a sufficiently long follow-up period before judgments are made about a disease that may, in the "wild", take 10 years to cause disease in $50 \%$ of subjects. The possibility remains that the strain has reduced pathogenicity and hence a longer 
time to progression, and that this can lead to the discovery of an even less pathogenic strain for vaccine use. However, data from primate models suggest that the pathogenicity of nef deletion strains varies. ${ }^{5}$ Other data show that the capacity for recombination, as well as mutation, in HIV and related viruses is very real. Thus it seems dangerous, on theoretical grounds, to rely on an assumption the the vaccine strain will remain non-pathogenic.

As more data emerge therefore, the validity of the concept of an identifiable stable attenuated strain of HIV, rendering it suitable for vaccine use, is seen as premature. The data also underpin the need for very long term follow-up to validate any observational experience of wild-type variants before conclusions are drawn. This also highlights just how long the follow-up period of any trial would need to be before it could be judged safe. A vaccine safety trial (phase I) of the necessary ten to fifteen years' duration seems neither realistic, nor likely to be an acceptable pathway for those who would wish to stress the urgency. Yet how could one justify extrapolating from initial preliminary results of an apparently safe initial phase in order to proceed to large-scale phase II and III trials of efficacy, let alone safety? The Sidney cohort offers a brutal reminder of the dangers of reading the results too soon.

The balance of these various strands of argument is difficult to resolve and different views can be readily justified. There is no question that the gauntlet thrown down to the scientific community by the volunteers for live attenuated vaccines has sharpened the debate on vaccine development. Indeed, it has lent other approaches some added urgency. The volunteers have also challenged ethicists to re-examine some of their basic concepts in a very different setting, and have enhanced our insights into the conduct of clinical trials.

Perhaps an almost overwhelming argument against attempting such trials with the present state of knowledge, is that there is a real risk that a truly pathogenic strain could turn out to have been used, or could emerge over time, in the vaccinees. If that were to happen, even in one recipi- ent, it would almost certainly mean that the live attenuated approach could never be tried again. So to proceed with this adventurous approach, even with willing volunteers, would probably jeopardise the possible validity of the concept and a potentially valuable approach to HIV vaccine development for all time. That heavy burden of responsibility suggests that the challenge should not be taken up at this time. Rather, the validity of the concept should be recognised and developed further in preclinical settings. The critical risk of the premature application of this approach, even in willing and altruistic volunteers, is not just the risk to the individuals, which they knowingly accept, but the significant threat to a whole strand of future investigation and application. The challenge has, however, facilitated an acceptance of the legitimacy of the concept of a live attenuated vaccine approach, which in itself is an achievement.

\section{Author's note}

This paper is based on a talk given at a conference organised by the Royal Free Centre for HIV medicine at the Royal College of Physicians, London in October 1998.

Anthony 7 Pinching is Louis Freedman Professor of Immunology and Fellow, Department of Human Science and Medical Ethics, St Bartholomew's and The Royal London School of Medicine and Dentistry, Queen Mary \& Westfield College, West Smithfield, London EC1A $7 B E$.

\section{References}

1 Guenter D, Esparza A, Macklin R. Ethical considerations in international HIV vaccine trials: summary of a consultative process conducted by the foint United Nations Programme on HIVIAIDS (UNAIDS). Fournal of Medical Ethics 2000;26:37-43.

2 Beauchamp TL and Childress JF. Principles of biomedical ethics [4th ed]. Oxford: Oxford University Press, 1994.

3 Daniel MD, Kirchhoff F, Czajack SC, Sehgal PK, Desrosiers RD. Protective effects of a live attenuated SIV vaccine with a deletion in the nef gene. Science 1992;258:1938-41.

4 Learmont JC, Geczy AF, Mills J, Ashton LJ, Raynes-Greenow $\mathrm{CH}$, Garsia RJ, et al. Immunologic and virologic status after 14 to 18 years of infection with an attenuated strain of HIV-1. New England fournal of Medicine 1999;340:1715-22.

5 Ruprecht RM, Baba TW, Liska V. Attenuated HIV vaccine: caveats. Science 1996;271: 1790-2. 